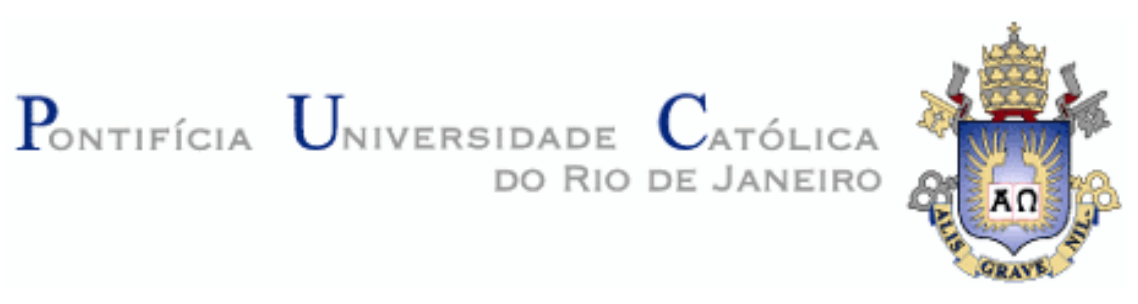

Barbara Maria Cabral Gouvea Coelho

\begin{abstract}
Estudo sobre a Viabilidade da Tecnologia PLC no Brasil e Considerações sobre uma Proposta de Tarifação
\end{abstract}

Dissertação de Mestrado

Dissertação apresentada como requisito parcial para obtenção do grau de Mestre pelo Programa de PósGraduação em Engenharia de Produção.

Orientador: Prof. Nélio Domingues Pizzolato Coorientador: Prof. Reinaldo Castro Souza 


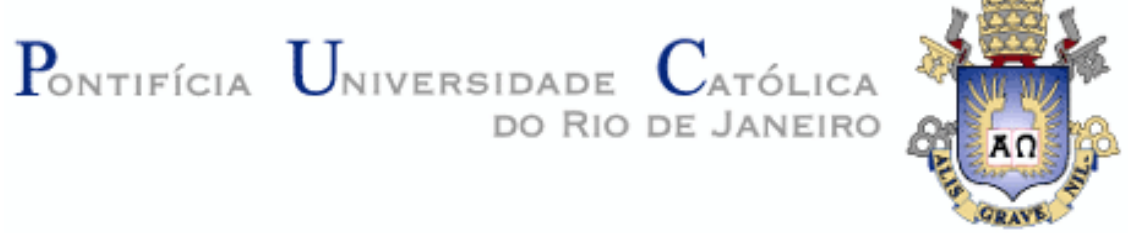

Barbara Maria Cabral Gouvea Coelho

\title{
Estudo sobre a Viabilidade da Tecnologia PLC no Brasil e Considerações sobre uma Proposta de Tarifação
}

Dissertação apresentada como requisito parcial para obtenção do grau de Mestre pelo Programa de PósGraduação em Engenharia de Produção da PUC-Rio. Aprovada pela Comissão Examinadora abaixo assinada.

\author{
Prof. Nélio Domingues Pizzolato \\ Orientador \\ Departamento de Engenharia Industrial - PUC-Rio \\ Prof. Reinaldo Castro Souza \\ Coorientador \\ Departamento de Engenharia Elétrica - PUC-Rio
}

Prof. José Francisco Moreira Pessanha Instituto de Matemática e Estatística - UERJ

Prof. João Carlos de Oliveira Aires Departamento de Engenharia Elétrica - Gama Filho

Prof. José Eugenio Leal

Coordenador Setorial do Centro Técnico Científico - PUC-Rio

Rio de Janeiro, 18 de Abril de 2012 
Todos os direitos reservados. É proibida a reprodução total ou parcial do trabalho sem autorização do autor, do orientador, do coorientador e da universidade.

\section{Barbara Maria Cabral Gouvea Coelho}

Graduou-se em Engenharia de Produção pela PUC-Rio em Dezembro de 2007. Em 2008 trabalhou como pesquisadora na consultoria de pesquisa e satisfação no IAG da PUC-Rio. Trabalhou como analista de Preços na Alvo Distribuidora de Combustíveis de 2008 a 2009.

Ficha Catalográfica

Coelho, Barbara Maria Cabral Gouvea

Estudo sobre a Viabilidade da Tecnologia PLC no Brasil e Considerações sobre uma Proposta de Tarifação / Barbara Maria Cabral Gouvea Coelho; orientador: Prof. Nélio Domingues Pizzolato; coorientador: Prof.Reinaldo Castro Souza - 2012.

113 f. : il. (color.) ; $30 \mathrm{~cm}$

Dissertação (mestrado) - Pontifícia Universidade Católica do Rio de Janeiro, Departamento de Engenharia Industrial, 2012. Inclui bibliografia

1. Engenharia Industrial - Teses. 2. Tecnologia PLC. 3. Regulamentação do PLC 4. Inovação. 5. Internet. 6. Energia Elétrica. 7. Estrutura Tarifária Elétrica. 8. Redes Inteligentes. I. Pizzolato, Nélio Domingues. II. Souza, Reinaldo Castro III. Pontifícia Universidade Católica do Rio de Janeiro. Departamento de Engenharia Industrial. IV. Título. 
Aos meus avós e a minha madrinha (in memoriam). A dedicação da autora. 


\section{Agradecimentos}

Agradeço primeiramente a Deus por toda inspiração e determinação, mesmo com inúmeros problemas que surgiram ao longo desta dissertação. Tudo se encaminhava para eu mudar de tema, mas com muita luta e colaboração de todos do Departamento da Industrial consegui concluir o tema original da dissertação.

A Casa Espírita Cristã Maria de Nazaré por todo apoio e equilíbrio emocional que me forneceram durante este período. Forças que fizeram que eu não desistisse da dissertação nem nos piores momentos.

Aos meus avós queridos, Elisa Panno, Odayl Maia Cabral, Moysés Coelho e Mariah Coelho. E a minha madrinha Vera Regina Panno. Apesar de não fazerem mais parte deste mundo estão sempre presentes em meu pensamento.

Aos meus pais, Sandra Coelho e Pedro Coelho, que sempre me apoiaram em todos os momentos da minha vida.

A todos os professores do Departamento da Industrial que sempre estavam dispostos a ajudar. Em especial ao meu orientador, Nélio Domingues Pizzolato, por ter assumido a minha orientação e ter solucionado problemas acadêmicos que surgiram ao longo da dissertação. E ao professor José Paulo Teixeira, pelos seus sábios conselhos.

A todos da secretaria do Departamento de Engenharia Industrial, especialmente à Cláudia e à Isabel, e aos secretários do professor Reinaldo Souza, Ana e Flávio, que estavam sempre dispostos a ajudar.

Um agradecimento em especial ao meu coorientador, professor Reinaldo Castro Souza, por toda a paciência e disponibilidade que mostrou no decorrer da dissertação. Agradeço por todas as fontes de informação, livros e especialistas no assunto PLC e na estrutura tarifária elétrica. Sua contribuição foi fundamental para o desenvolvimento deste trabalho, sem a sua ajuda este trabalho não seria possível. Agradeço por ele ter aceitado me coorientar mesmo eu sendo de outro departamento.

Ao CNPq, pelo suporte financeiro que possibilitou a execução deste trabalho.

A PUC-Rio, por fazer parte da minha formação e por permitir que eu tenha vivenciado os melhores anos da minha vida, conhecendo pessoas maravilhosas nesta instituição. É um orgulho que ela faça parte da minha vida pessoal e profissional desde a graduação e agora ter retornado a mim com o mestrado.

Ao Bennett por ceder a sua biblioteca para frequentadores que não fazem parte da sua instituição de ensino.

Agradeço pelos amigos verdadeiros que fiz ao longo do mestrado, em especial a Luíz Emídio.

O agradecimento sincero da autora. 


\section{Resumo}

Coelho, Barbara Maria Cabral Gouvea Coelho; Domingues Pizzolato, Nélio; Castro Souza, Reinaldo; Estudo sobre a Viabilidade da Tecnologia PLC no Brasil e Considerações sobre uma Proposta de Tarifação. Rio de Janeiro, 2012. 113p. Dissertação de Mestrado Departamento de Engenharia Industrial, Pontifícia Universidade Católica do Rio de Janeiro.

O PLC, Power Line Communication, é uma tecnologia inovadora que está sendo utilizada para a transmissão de dados e voz via rede elétrica. Esta tecnologia se encontra na fase de testes na maioria dos países e o seu amadurecimento no mercado está previsto a partir do ano de 2016, segundo especialistas e pesquisas na área. Como é um produto novo, em fase de lançamento, não é possível mensurar a dimensão que terá no mercado, ou seja, a sua participação no mercado da internet banda larga. A proposta da tecnologia é tornar-se mais uma opção de banda larga para o consumidor, concorrendo com os atuais serviços via cabo e rede sem fio. As empresas de energia elétrica levam vantagem por já terem a infraestrutura pronta para implementar o PLC, no entanto serão necessárias algumas adaptações na rede elétrica. A difusão do PLC no mercado depende não só do avanço da tecnologia como de uma reestruturação do setor elétrico brasileiro. Outra barreira encontrada pela tecnologia são os diversos padrões existentes pois não existe uma regulamentação universal, o que dificulta a importação de equipamentos, por exemplo. Hoje no Brasil, as empresas de energia e de telecomunicações estudam meios para se implementar a tecnologia PLC de forma a se construir um modelo de negócios que seja viável e rentável para elas.

\section{Palavras-Chave}

Tecnologia PLC; Regulamentação do PLC; Inovação; Internet; Energia Elétrica; Estrutura Tarifária Elétrica; Redes Inteligentes. 


\section{Abstract}

Coelho, Barbara Maria Cabral Gouvea; Domingues Pizzolato, Nélio (Advisor); Castro Souza, Reinaldo (Coadvisor). Study on the Viability of PLC Technology in Brazil and Considerations for a Proposed Pricing. Rio de Janeiro, 2012, 113p. MSc. Dissertation - Departamento de Engenharia Industrial, Pontifical Catholic University of Rio de Janeiro.

The PLC, Power Line Communication, is an innovative technology that is being used to transmit voice and data over power line. This technology is in testing phase in most countries and its maturity in the market is predicted from the year 2016 according to experts and researchers in the area. As a new product being launched, it is not possible to measure the extent it will have on the market, i.e. the future market share of broadband internet. The proposed technology is becoming an alternative choice of broadband to consumers in competition with existing services via cable and wireless network. The energy companies have the advantage of having already the infrastructure ready to implement the PLC, but some adjustments will be required in the grid. The spread of the PLC market depends not only on the advancement of technology but also in the restructuring of the Brazilian electric sector. Another technology barrier are the various existing standards because there are no universal regulations, which can make the importation of equipment a difficult task, for example. Today in Brazil, energy and telecommunications companies are studying ways to implement the PLC technology in order to build a business model that is viable and profitable for them.

\section{Keywords}

PLC Technology; PLC Regulation; Inovation; Internet; Electrical Energy; Electrical Pricing Structure; Smart Grid. 


\section{Sumário}

$1 \quad$ Introdução 15

1.1 Apresentação do Problema 16

$\begin{array}{lll}1.2 & \text { Objetivo da Dissertação } & 17\end{array}$

$\begin{array}{lll}1.3 & 17\end{array}$

1.4 Delimitação 18

1.5 Contexto do Tema 18

$\begin{array}{lll}1.6 & \text { Estrutura da Dissertação } & 19\end{array}$

2 Panorama do PLC 21

$\begin{array}{lll}2.1 & \text { Histórico } & 21\end{array}$

2.2 Aplicações 22

2.2.1 Avanço da Tecnologia 23

2.2.2 Funcionamento do PLC 24

$2.3 \quad$ Aspectos Regulamentares 27

2.4 Principais Tecnologias PLC 28

2.4.1 X-10 PLC (Power Line Carrier) 29

2.4.2 Intellon CEBus 29

2.4.3 Echelon Lon Works 29

2.4.4 Adaptive Networks 29

$\begin{array}{lll}2.4 .5 & \text { PLUG-IN } & 30\end{array}$

2.4.6 HomePlug 30

$\begin{array}{lll}2.4 .7 & \text { DS2 } & 30\end{array}$

$3 \quad$ Estudos do PLC 31

3.1 Projetos no Mundo 33

3.1.1 Projeto OPERA 39

3.1.2 Outros Investimentos e Projetos na Tecnologia PLC 40

3.2 Projetos no Brasil 41

3.2.1 Projeto Vilas Digitais 45

$\begin{array}{lll}\text { 3.2.2 } & \text { Projeto Piloto de Empresas } & 47\end{array}$ 
3.2.3 Projeto PNBL 48

$4 \quad$ Motivação para as Empresas de Energia 52

4.1 Vantagens 53

4.2 Desvantagens 55

5 Smart Grid 58

5.1 Relação do PLC com o Smart Grid 66

6 Tarifação da Energia Elétrica 69

6.1 Nova Tarifação da Energia Elétrica 79

6.2 Estrutura da Rede Elétrica 85

$7 \quad$ Modelos de Négocio para o PLC 87

7.1 Considerações sobre uma Possível Tarifação do PLC 88

8 Conclusão 93

Referências Bibliográficas $\quad 95$ 


\section{Lista de Figuras}

Figura 1: Funcionamento de uma rede de PLC 25

Figura 2: Exemplo de adaptadores para eliminar fios $\quad 25$

Figura 3 : Mapa Comparativo PLC Brasil X PLC Mundo 32

Figura 4: Portfólio tecnológico estratégico da aplicação "serviços convergentes de telecomunicações" no Brasil (2008-2025) 33

Figura 5: Funcionamento de uma casa inteligente 65

Figura 6: Quanto se paga por componente em uma conta de luz de $\mathrm{R} \$ 100,00$ (média / Brasil 2007)

Figura 7: Valor vigente das tarifas residenciais em 13 de março de 2012 


\section{Lista de Tabelas}

Tabela 1: Organizações atuantes no Mundo para PLC 34

Tabela 1 continuação: Organizações atuantes no Mundo para PLC 35

Tabela 1 continuação: Organizações atuantes no Mundo para PLC 36

Tabela 1 continuação: Organizações atuantes no Mundo para PLC 37

Tabela 1 continuação: Organizações atuantes no Mundo para PLC 38

Tabela 2: Número de usuários de PLC em 15 países 38

Tabela 3: Organizações atuantes no Brasil para PLC 41

Tabela 3 continuação: Organizações atuantes no Brasil para PLC 42

Tabela 3 continuação: Organizações atuantes no Brasil para PLC 43

Tabela 3 continuação: Organizações atuantes no Brasil para PLC 44

Tabela 3 continuação: Organizações atuantes no Brasil para PLC 45

Tabela 4: Principais encargos setoriais com a contribuição do consumidor em $2006 \quad 71$

Tabela 5: Tributos presentes na conta de luz 72

Tabela 6: Subclassificação do Grupo A 74

Tabela 7: Sub-classificação do Grupo B 74

Tabela 8: Estrutura da tarifação horossazonal azul 77

Tabela 9: Estrutura da tarifação horossazonal verde 78 


\section{Lista de Siglas e Abreviaturas}

ABDI - Agência Brasileira de Desenvolvimento Industrial

ADSL - Assymmetric Digital Subscriber Line

ANATEL - Agência Nacional de Telecomunicações

ANEEL - Agência Nacional de Energia Elétrica

APTEL - Associação de Empresas Proprietárias de Infraestrutura e

Sistemas Privados de Telecomunicações

ARRL - American Radio Relay League

BNDES - Banco Nacional de Desenvolvimento Econômico e Social

BPL - Broadband Over Powelines

CEBus - Consumer Electronic Bus

CEEE - Companhia Estadual de Energia Elétrica

CELG - Companhia Energética de Goiás

CEMAR - Companhia Energética do Maranhão

CEMIG - Companhia Energética de Minas Gerais

CETA-SENAI - Centro de Excelência em Tecnologias Avançadas SENAI

(RS)

CIP - Contribuição de lluminação Pública

COFINS - Contribuição para o Financiamento da Seguridade Social

COPEL - Companhia Paranaense de Energia

CTBC - Companhia de Telecomunicações do Brasil Central

DNAEE - Departamento Nacional de Águas e Energia Elétrica

DS2 - Diseños de Silicio

DSSS - Direct Sequency Spread

EDF - Électricité de France

EIA - Associação das Indústrias de Eletrônica

ESS_SE - Encargo de Serviços do Sistema por Segurança Energética

FCC - Federal Communications Comission

FITEC - Centro de Inovação Tecnológica

FSK - Frequency Shift Keying

GESAC - Governo Eletrônico Serviço de Atendimento ao Cidadão

HD - Hard Disk 
HD-PLC - High-Definition Power Line Communication

IBGE - Instituto Brasileiro de Geografia e Estatística

IBM - International Business Machines Corporation

ICMS - Imposto sobre Circulação de Mercadorias Serviços

IDH - Índice de Desenvolvimento Humano

IEEE - Institute of Electrical and Electronic Engineers

IRF - Interferência de Rádio Frequência

LON - Local Operating Network

OEM - Original Equipment Manufacturer

OFDM - Orthogonal Frequency Division Multiplexing

OPERA - Open PLC European Research Alliance

P\&D - Projeto e Desenvolvimento

PIS - Programa de Integração Social

PLC - Power Line Communication

PLD - Preço de Liqquidação de Diferenças

PNAD - Pesquisa Nacional por Amostra de Domicílios

PNBL - Plano Nacional de Banda Larga

PROCEL - Programa Nacional de Conservação de Energia Elétrica

Procempa - Companhia de Processamentos de Dados de Porto Alegre

SEBRAE - Serviço Brasileiro de Apoio às Micro e Pequenas Empresas

Sercomtel - Operadora de Telecomunicações em Londrina - Paraná

Telebrás - Telecomunicações Brasileiras S. A.

Telebrasil - Associação Brasileira de Telecomunicações

TST - Tecnologia e Sistemas de Telecomunicações Ltda.

UFRGS - Universidade Federal do Rio Grande do Sul

UPA - Universal Powerline Association

USP - Universidade de São Paulo

VolP - Voice Over IP

VSAT - Very Small Aperture Terminal

Wi-Fi - Wireless Fidelity

X-10 PLC - Power Line Carrier 
"Para obter algo que você nunca teve, precisa fazer algo que nunca fez".

Chico Xavier 